\title{
A New Neural Network-Based Intrusion Detection System for Detecting Malicious Nodes in WSNs
}

\author{
1Narmatha C \\ ${ }^{1}$ Department of Computer Science, Faculty of Computers and Information Technology, University of Tabuk, Tabuk City, \\ Saudi Arabia. \\ "Corresponding Author: cmnarmath@gmail.com and narmatha@ut.edu.sa
}

Received: 02.09 .2020 , Revised: 05.12 .2020 Accepted: 15.12.2020, Published: 22.12.2020

DOI:

10.53409/mnaa.jcsit20201301
Abstract: The Wireless Sensor Networks (WSNs) are vulnerable to numerous security hazards that could affect the entire network performance, which could lead to catastrophic problems such as a denial of service attacks (DoS). The WSNs cannot protect these types of attacks by key management protocols, authentication protocols, and protected routing. A solution to this issue is the intrusion detection system (IDS). It evaluates the network with adequate data obtained and detects the sensor node(s) abnormal behavior. For this work, it is proposed to use the intrusion detection system (IDS), which recognizes automated attacks by WSNs. This IDS uses an improved LEACH protocol clusterbased architecture designed to reduce the energy consumption of the sensor nodes. In combination with the Multilayer Perceptron Neural Network, which includes the Feed Forward Neutral Network (FFNN) and the Backpropagation Neural Network (BPNN), IDS is based on fuzzy rule-set anomaly and abuse detection based learning methods based on the fugitive logic sensor to monitor hello, wormhole and SYBIL attacks.

Keywords: Magnetic Resonance Imaging, Brain tumor, and Deep Belief Network.

\section{INTRODUCTION}

ireless sensor networks (WSNs) are W current technology, and researchers have attracted a lot of attention. Usually, the low power and low-cost environment of the WSN includes a large number of sensors that are arbitrarily distributed or reassigned manually over the target location. Due to its potential features and applications such as healthcare, monitoring, domestic uses, surveying systems, and disaster management [1], wireless sensor networks are becoming a powerful and familiar technology. There are poor communication, calculation, and energy capacities for wireless sensor nodes. Broadcast messages are a useful and essential prototype in wireless sensor networks, which permit multiple users to efficiently combine and distribute Message Packages across their network to obtain their data of interest. Figure 1 shows an example diagram for WSN [2].

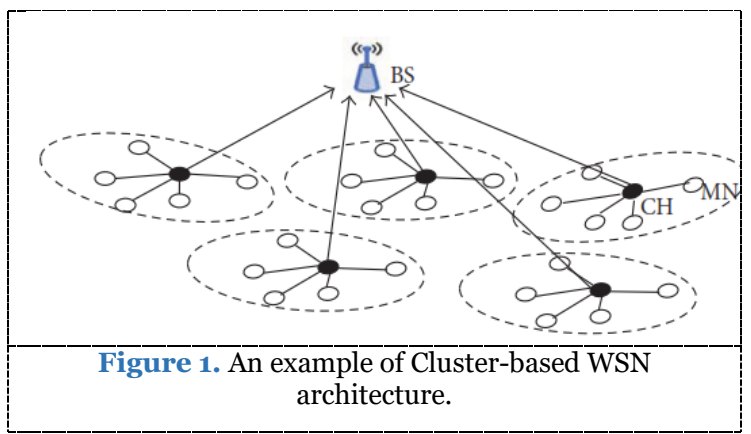

Data can be transmitted over long distances via intermediate nodes as WSNs are susceptible to both internal and external outbreaks. More generally, due to their resource-restricted nature [3], they cannot deal with a hard attacker. In this situation, it is necessary to protect the system from the attackers by a secondary stage of protection, called the intrusion detection system (IDS). Efficient IDS [4] can identify the extensive attack technologies employed by the attackers.

Unfortunately, most sensor networks can be highly susceptible to attack due to WSN 
features, and antagonists can probably create network traffic that can also cause a massive drop of packets or change the original message content of packets [5]. To ensure secure communication between nodes, authentication strategies are incorporated on the network. In WSNs, the safe transmission of data between nodes is significant.

In this work, IDS uses a multilayer neural perceptron system to detect the anomaly and to detect a misuse based upon the fuzzy rules. Also, the feed forward neural network is used to integrate the results of the detection and to signify the various kinds of attackers (similar to Sybil, the wormhole attack, and the hello flood attack).

Data transmission in longer distances can be performed through intermediate nodes since WSNs are vulnerable to internal and external outbreaks. Most commonly, they cannot handle a fierce attacker owing to their resourcerestricted nature [3]. In this condition, a secondary stage of defence mostly called the Intrusion Detection System (IDS), is needed to protect the system from the attackers. The vast attacking techniques developed by the attackers can be detected by making use of efficient IDS [4].

Unfortunately, the majority of the sensor networks are susceptible to attacks because of WSN characteristics, and antagonists can create network traffic, which can also cause massive packet drop during the broadcasting of the packets or change the original content of the message in the packets [5]. Thus, authentication strategies are implemented in the network to ensure secure communication between the nodes. In WSNs, it is essential to carry out secure data transmission between the nodes.

In this work, IDS uses anomaly detection and misuse detection based on fuzzy rule sets along with the Multilayer Perceptron Neural Network. The Feed Forward Neural Network, along with the Backpropagation Neural Network, is utilized to integrate the detection results and indicate the different types of attackers (i.e., Sybil attack, wormhole attack, and hello flood attack).

The rest of the paper is organized as follows. Section 2 deals with the related work on IDS methods in WSN. Section 3 describes the methods proposed for defining the MRI brain tumor classification with DBN. Section 4 discusses the experimental findings. Section 5 includes the conclusion and prospective work.

\section{RELATED WORK}

For the securement of wireless sensor networks in routing attacks, a new intrusion detection framework is proposed in [6]. The proposed method focuses on the neighboring nodes in a distributed environment to identify intrusions.

A lightweight, energy-efficient system proposed in [7] that uses mobile agents for metrically detecting intrusions based on sensor nodes energy consumption. To predict energy consumption, a linear regression model is applied.

An IDS framework is inspired by the HIV system that can be used in the network of wireless sensors [8]. The Dendritic cell algorithm uses an improved decentralized, and personalized version enables nodes to monitor their area and work together to identify an intrusive.

A WSN intrusion-detection system based on the number of active, successful deliveries is being proposed to trust-based adaptive tracking acknowledgment (TRAACK) and the Kalman filter to anticipate node trust in [9]. A novel trust management scheme based on the theory of Dempster - Shafer evidence for malicious node detection is proposed to deal with the situation of the quantification and uncertainty of trust [10].

In [11], the trust system for physical sensor networks (WSNs) on the physical layer has been proposed for intrusion detection based on physical layouts (PL-IDS). In [12], the WSNs propose a game theory-based multi-level detection frame. A mixture of specification rules and a lightweight anomaly detection module based on the neural network is applied to the proposed framework for identifying malicious sensor nodes. Calculation and energy-intensive, which negatively impact on the overall lifetime of the WSNs, are the most current frameworks for the intruder detection of wireless sensor networks (WSNs). This work consequently proposes IDS for neural network detection of different attacks in WSN.

\section{PROPOSED METHODOLOGY}

The work being proposed is intended to detect hello flooding, wormhole, and Sybil attacks in the WSN with the IDS. To recognize attackers of different types, use an enhanced LEACH protocol (with fuzzy rules). For the detection of these attacks, IDS benefits from both anomaly detection and misuse detection models. A higher detection rate and a low positive rate may be achieved with the proposed IDS. In the meantime, MPNN's machinelearning strategy can discover, which included new instances practically by enduring unknown 
attacks. The architecture diagram of IDS using MPNN is shown in Figure 1.

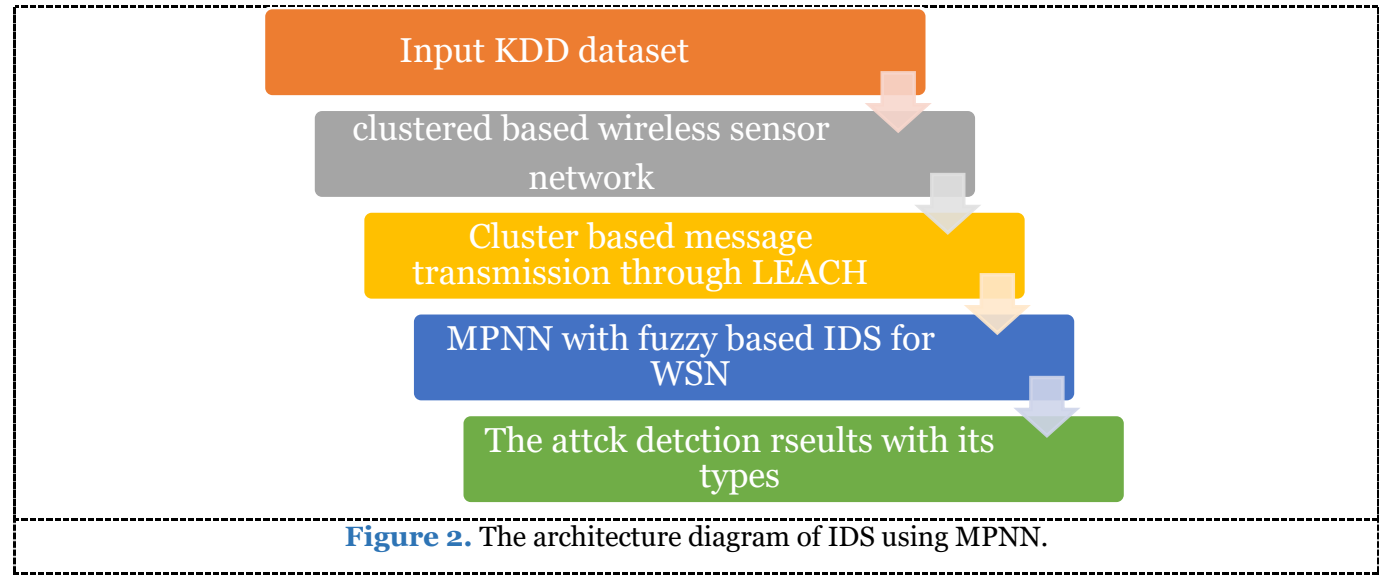

\subsection{KDD Dataset Description}

The cup KDD-99 is the most common dataset for algorithm training. It is the DARPA-98 dataset sub-set. Dataset KDD-99 is a multiform dataset. There are 4.8 million cases in this dataset. Categorical and integer characteristics of attributes are used in the dataset, and it has 42 features [12].

\subsection{Multilayer Perceptron Neural Network (MPNN)}

The model MPNN is divided into FFNN and BPNN and is used to estimate the accuracy of the detection of the three different attackers. Because of the development of advanced technology, attackers improve with each day, and thus there is a great need for the improvement of existing IDS and system capacity. The IDS is an advanced intelligent detection system to overcome such problems. The mechanism for the machine learning mechanism can identify and learn new types of attacks. However, the data packets cannot be correctly sorted by an unknown assault by the detection model. Even farther, such data packets would also be transferred to the MPNN so that the detection system can understand and introduce the new type of attack detection system. Figure 2 provides the suggested MPNN methodology with fuzzy IDS in the WSN.

This is where the MPNN is going to develop the FFNN and BPNN IDS mechanism since these neutral networks could maintain the stability of the system with a considerable number of data and can listen to various types of attackers. FFNN would, however, make headway with detection and simultaneously estimate the new types of attack. The proposed BPNN will use the MPNN supervised learning mechanism to cluster unknown attacks that incorporate an input layer, a hidden layer, and an output layer. In the detection of excesses, a scheme cannot detect accurate data packet attacks since the layer, and the input nodes of data packets are selected by using the features selected. In the beginning, the stage is the number of output nodes. Therefore, the proposed fuzzy-based MPNN would be used to create different kinds of clusters. Thus, the results of the output nodes are improved when each output node sets a new type of detection method. An artificially supervised learning mechanism will be used to estimate the corresponding points for each output result in every data package of unknown attackers. The results of the output node will later be detected to determine the value of the output node.

When this value is higher than the alert value, then the packets inserted correspond to the output node; therefore, it is cluster-referred, and MPNN must simply modify the weights. However, if the respective output node value is much less than the warning value, the data packet is not the same as the connected weight. It therefore does not match this cluster. This data packet is not the same in the same way. The next winning node results must be found to see whether the alertness test can pass or else it will introduce a unique output node that will identify a new attack.

In addition, the sample data through the experimental simulation will be examined to obtain the true vigilance value. The information about a cluster is contained in the MPNN of the misuse detection scheme to include fresh detection classes, whereas the cluster-member values acquire the defined threshold. 


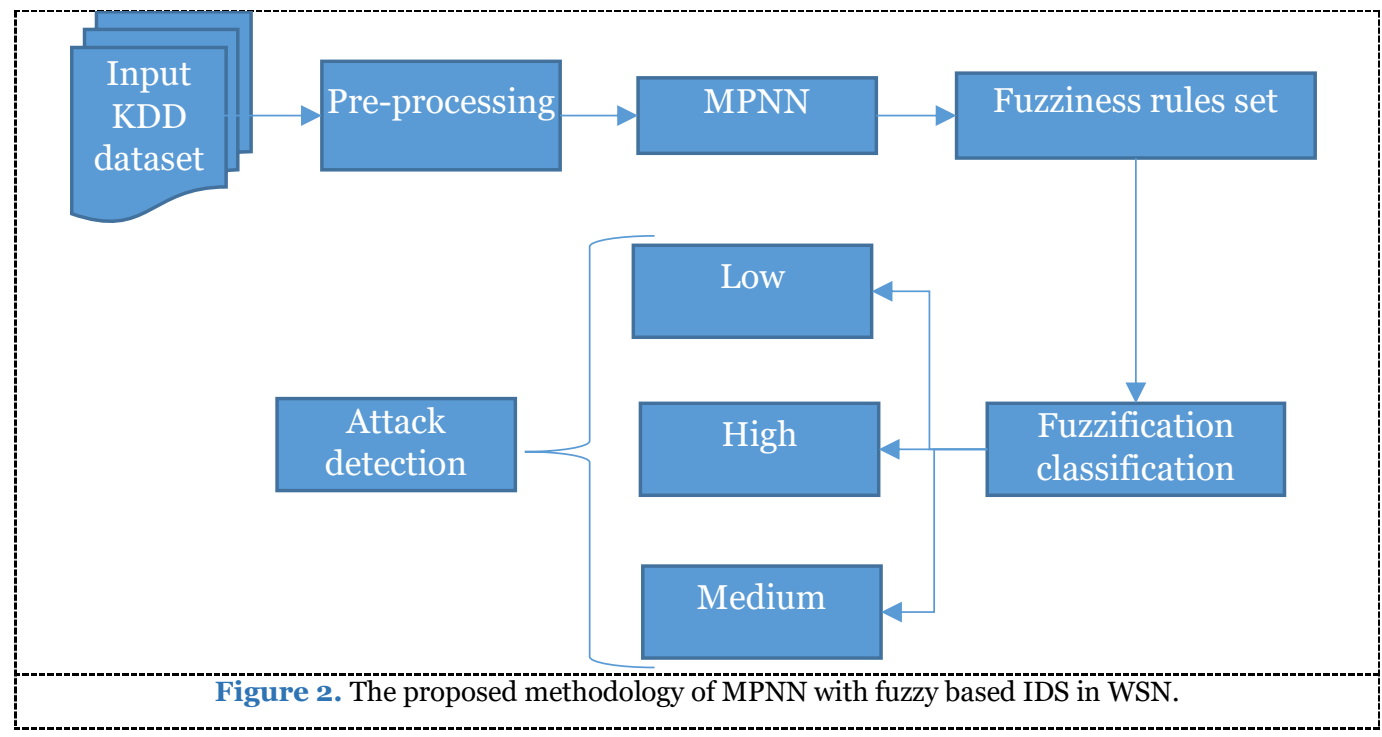

Fuzzy Rules Set Based Multilayer Perceptron Neural Network: The data are trained using supervised FFNN classifier by applying the $n$ hidden nodes from the specified dataset of labelled examples, a dataset of unlabelled examples, and a test dataset. In order to get the final output as the sigmoid activation algorithm, the hidden node is implemented with BPNN supervised classification. Then, as membership vector MV is achieved on each unlabeled sample by analysing US application of MPNN supervised learning system, the membership vector of each unlabeled US sample generated during this process is further applied by using equation (1) to obtain the fuzziness F(MV):

$$
\begin{aligned}
& F(M V)=-\frac{1}{n} \sum_{i=1}^{n}\left(\mu_{\mathrm{i}} \log \mu_{\mathrm{i}}+\left(1-\mu_{\mathrm{i}}\right) \log (1-\right. \\
& \left.\left.\mu_{\mathrm{i}}\right)\right) * e r_{i} \\
& e r_{i}=d o_{i}-a o_{i}
\end{aligned}
$$

where $M V=\left\{\mu_{1}, \mu_{2}, \ldots, \mu_{n}\right\}$ is a fuzzy set and the $e r_{i}$ is the error rate, $d o_{i}$ signifies the desired output and $a o_{i}$ denotes the actual output which resulted from the MPNN. Furthermore, the Fuzziness value is categorized into three distinct groups: low, high, and medium. Samples that indicate high fuzziness and low fuzziness classes are collected, and these classes are additionally included with training darta to get a revised dataset labeled as new training data to train the FFNN and check it with BPNN.

\subsection{Fuzzy with MPNN Based IDS Model for malicious node detection}

Methods of anomaly detection and misuse detection use many methodologies of wellestablished behaviors of attack, therefore build the latest strategy to resolve or protect against these behaviors of attacks [13]. Most intrusion detection techniques promise through the training data to detect the attacks, but they fail uncertainly. The proposed analysis is based on MPNN, consisting of
FFNN along with BPNN, and is implemented in this study to have the highest detection rate in the supervised approach to learning. The proposed approach showed figures outside of the traditional relationship between variables of input and output, so this fits the traditional mass. For achieve the greatest accuracy, it will minimize the error rate that exists in the code. Researchers therefore suggested fuzzy-based FFNN and BPNN to achieve the highest degree of accuracy in the detection of attacks by massive training for clustered AHIDS.

After the integration of the training data into the BPNN, can compare the actual performance results via the FFNN process. The error and rectification value of both hidden and output layers is determined by the back propagation process in the MPNN. To change the biases and weights of the networks, this length is called the epoch when all the training data have been used. The training data will be constantly discovered and periodically arranged with the aid of the epochs, the weights according to the layers, unless the output layer value is the same as the target value and then the training data is completed. Full irregular packets are then detected by the anomaly detection system, and then forwarded to the system for misuse for further verification.

Next, add the pre-processing step to cover up the irregular packets into a binary value, and then forward the binary value to the output value calculation scheme for misuse detection. Eventually, to get the best integration, the product of the detection value is provided to the fuzzy module with MPNN model. The fuzzy module is used to allow the best decision taking to classify the attackers and their different forms of attack by combining anomaly detection scheme and detection module for misuse. Through applying the rules to combine the outputs of the two detection schemes, the fuzzy rule-based method is used to support the decision-making model, and the main benefit of this 
study is to obtain fast and reliable results. Tabulation gives the fuzzy dependent rules. This mechanism operates using a fuzzy logic controller; first, the input parameters (FPNN) are assigned to the Fuzzy Inference Method (FIS) in the fuzzification process. The FIS performs on the basis of the Fuzzy membership (triangular) and the Fuzzy rule that is applied to the input parameters to determine the correct fuzziness to identify the concentrations of attackers. Therefore, in FIS, these parameters are analyzed which checks the fuzzy rules and functions to produce the results to defuzzify where the output parameters are extracted as low (Sybil attack), mild (wormhole attack), and high (hello flooding attack).

\section{EXPERIMENTAL RESULTS AND DISCUSSION}

The performance of the proposed MPNN with fuzzy is evaluated in this section, and the performance results are compared with existing TRAACK [9], PL-IDS [11] and lightweight neural network [12] schemes. The performance measurement is done in terms of precision, fmeasurement, recall, accuracy and attacker detecetion rate. Here estimate the different types of attackers such as hello flooding, wormhole, and Sybil attacks.

Precision: It reflects the proportion of positive samples correctly classified as expected in equation (3):

$$
\text { Precision }=\frac{T P}{F P+T P}
$$

Recall: The recall of a classifier reflects the positive samples properly assigned to the total number of positive samples and is calculated as in equation (4):

$$
\text { Recall }=\frac{T P}{T P+F N}
$$

F-measure: this is also referred to as $\mathrm{F}$ 1-score, and as in equation (5) is the harmonic mean of precision and recall:

$$
F-\text { measure }=\frac{2 *(\text { Recall } * \text { Precision })}{(\text { Recall }+ \text { Precision })}
$$

Accuracy: This is one of the most frequently used performance classification measures and is defined as a ratio between the correctly classified samples and the total number of samples as in equation (6):

$$
\text { Accuracy }=\frac{T P+T N}{T P+T N+F P+F N}
$$

Attacker detection rate: It is calculated using equation (7)

$$
\text { Attacker detection rate }=\sum_{i=2}^{c} \frac{T P_{i}}{T P_{i}+F N_{i}} .
$$

Where, $c$ is the number of classes, true positive $\left(T P_{i}\right)$ samples are properly classified as no attacker of the $i$ th class, false positive (FP) samples are incorrectly classified as attacker, True negative (TN) samples are properly classified as attacker, and false negatives $\left(F N_{i}\right)$ are incorrectly classified as attacker of the $i$ th class.

\subsection{Precision Rate comparison}

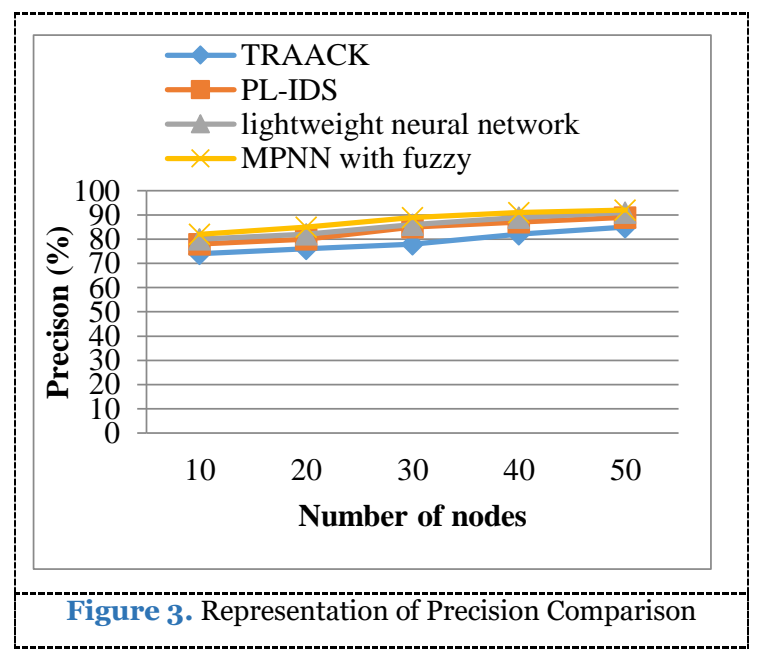

From the above Figure 4, the graph shows how accurate the number of images in the specified datasets is compared. These methods are implemented TRAACK, PL-IDS, lightweight neural network and MPNN with fuzzy. When the number of records increases according to the precision value, from this graph, it is learned that the proposed MPNN with fuzzy offers 92\% higher precision than previous methods that yield better results in the classification of attackers. The numerical results of Precision Comparison is shown in Table 1.

Table 1. The numerical results of Precision Comparison

\begin{tabular}{ccccc}
\hline $\begin{array}{c}\text { Number } \\
\text { of nodes }\end{array}$ & TRAACK & $\begin{array}{c}\text { PL- } \\
\text { IDS }\end{array}$ & $\begin{array}{c}\text { lightweight } \\
\text { neural } \\
\text { network }\end{array}$ & $\begin{array}{c}\text { MPNN } \\
\text { with } \\
\text { fuzzy }\end{array}$ \\
\hline 10 & 74 & 78 & 80 & 82 \\
\hline 20 & 76 & 80 & 82 & 85 \\
\hline 30 & 78 & 85 & 86 & 89 \\
\hline 40 & 82 & 87 & 89 & 91 \\
\hline 50 & 85 & 89 & 91 & 92 \\
\hline
\end{tabular}

\subsection{Recall comparison}

From the above Figure 5, the graph illustrates the recall relation for the number of images in the listed datasets. These methods are implemented as TRAACK, PL-IDS, lightweight neural network and MPNN with fuzzy. Increasing the number of images often increases the correct value for the recall. Through this graph, it is discovered that the current MPNN with fuzzy offers recall 95\% higher than previous methods. 


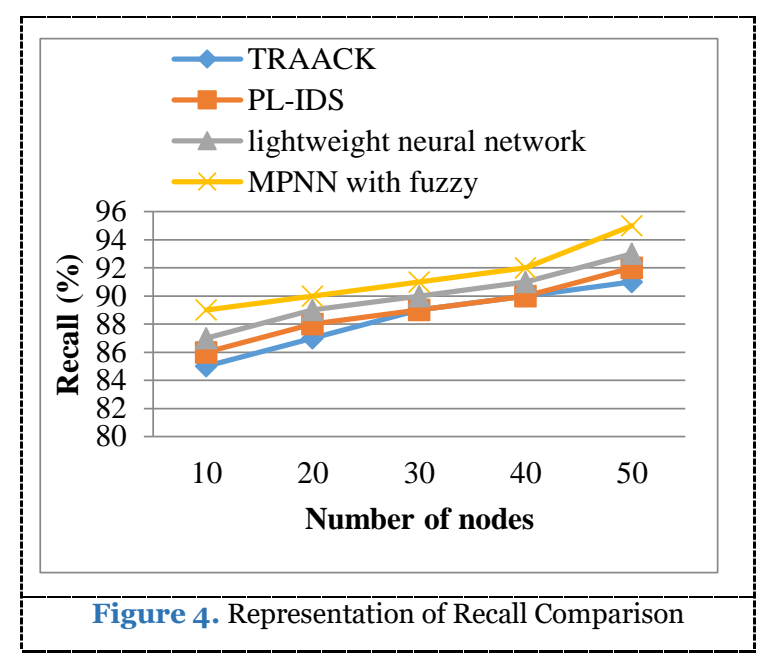

The explanation for this is that the MPNN with fuzzy classifies the fuzziness, which will enhance the detection and classification of attackers in WSN. The numerical results of Recall Comparison is shown in Table 2.

Table 2. The numerical results of Recall Comparison

\begin{tabular}{ccccc}
\hline $\begin{array}{c}\text { Number } \\
\text { of nodes }\end{array}$ & TRAACK & $\begin{array}{c}\text { PL- } \\
\text { IDS }\end{array}$ & $\begin{array}{c}\text { lightweight } \\
\text { neural } \\
\text { network }\end{array}$ & $\begin{array}{c}\text { MPNN } \\
\text { with } \\
\text { fuzzy }\end{array}$ \\
\hline 10 & 85 & 86 & 87 & 89 \\
\hline 20 & 87 & 88 & 89 & 90 \\
\hline 30 & 89 & 89 & 90 & 91 \\
\hline 40 & 90 & 90 & 91 & 92 \\
\hline 50 & 91 & 92 & 93 & 95 \\
\hline
\end{tabular}

\subsection{F-measure Rate comparison}

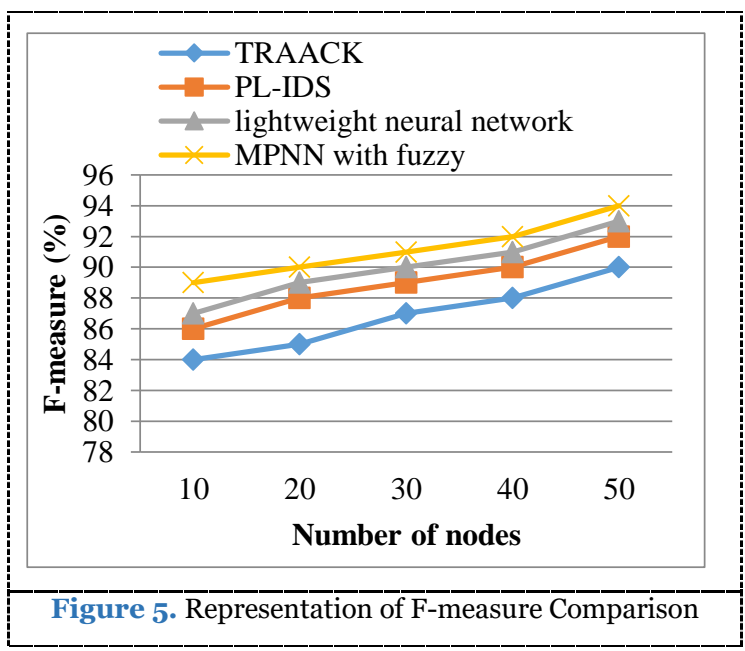

From the above Figure 6, the graph explains the fmeasure relation for the number of images in the given datasets. These methods are implemented as TRAACK, PL-IDS, lightweight neural network and MPNN with fuzzy. When the number of data is increased, and the f-measure value is increased accordingly. From this graph it is learned that the proposed MPNN with fuzzy offers 95\% higher f- measurement than previous methods. Therefore the proposed MPNN with fuzzy algorithm is stronger than the current algorithms in terms of better performance of classifying attackers in WSN. The numerical results of F-measure Comparison is shown in Table 3.

Table 3. The numerical results of F-measure Comparison

\begin{tabular}{ccccc}
\hline $\begin{array}{c}\text { Number } \\
\text { of nodes }\end{array}$ & TRAACK & $\begin{array}{c}\text { PL- } \\
\text { IDS }\end{array}$ & $\begin{array}{c}\text { lightweight } \\
\text { neural } \\
\text { network }\end{array}$ & $\begin{array}{c}\text { MPNN } \\
\text { with } \\
\text { fuzzy }\end{array}$ \\
\hline 10 & 85 & 89 & 90 & 91 \\
\hline 20 & 87 & 90 & 91 & 92 \\
\hline 30 & 89 & 91 & 92 & 93 \\
\hline 40 & 90 & 92 & 93 & 94 \\
\hline 50 & 91 & 93 & 94 & 95 \\
\hline
\end{tabular}

4.4. Accuracy comparison

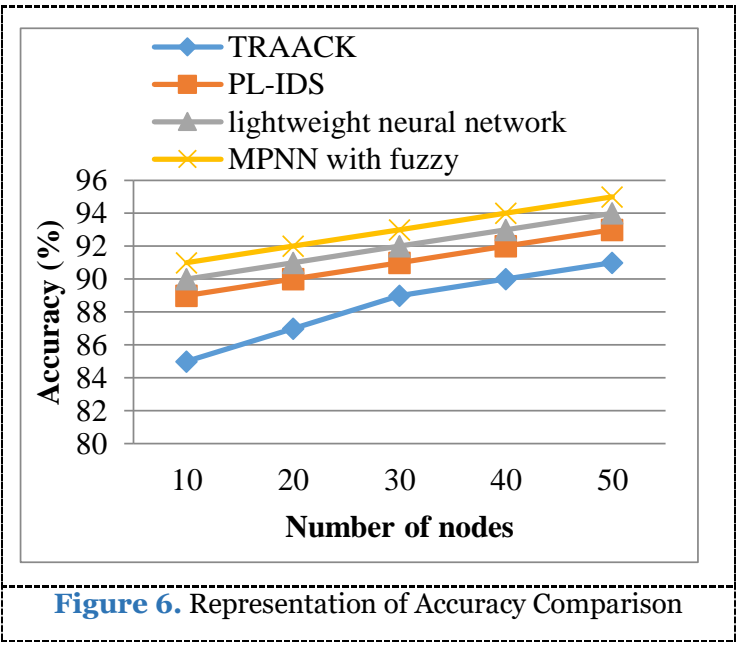

From the above Figure 7, the diagram illustrates the processing time relation for the number of images in the specified datasets. These methods are implemented as TRAACK, PL-IDS, lightweight neural network and MPNN with fuzzy.

From the graph, it is known that the proposed MPNN with fuzzy algorithm is higher than the existing algorithms with a high precision rate of $95 \%$ in terms of better attacking results. This is due to the fuzzy based extraction of the fuzziness in the MPNN algorithm, which increases the attacker classification results. The numerical results of Accuracy Comparison is shown in Table 4. 
Table 4. The numerical results of Accuracy Comparison

\begin{tabular}{ccccc}
\hline $\begin{array}{c}\text { Number } \\
\text { of nodes }\end{array}$ & TRAACK & $\begin{array}{c}\text { PL- } \\
\text { IDS }\end{array}$ & $\begin{array}{c}\text { lightweight } \\
\text { neural } \\
\text { network }\end{array}$ & $\begin{array}{c}\text { MPNN } \\
\text { with } \\
\text { fuzzy }\end{array}$ \\
\hline 10 & 85 & 89 & 90 & 91 \\
\hline 20 & 87 & 90 & 91 & 92 \\
\hline 30 & 89 & 91 & 92 & 93 \\
\hline 40 & 90 & 92 & 93 & 94 \\
\hline 50 & 91 & 93 & 94 & 95 \\
\hline
\end{tabular}

\subsection{Attacker detection rate comparison}

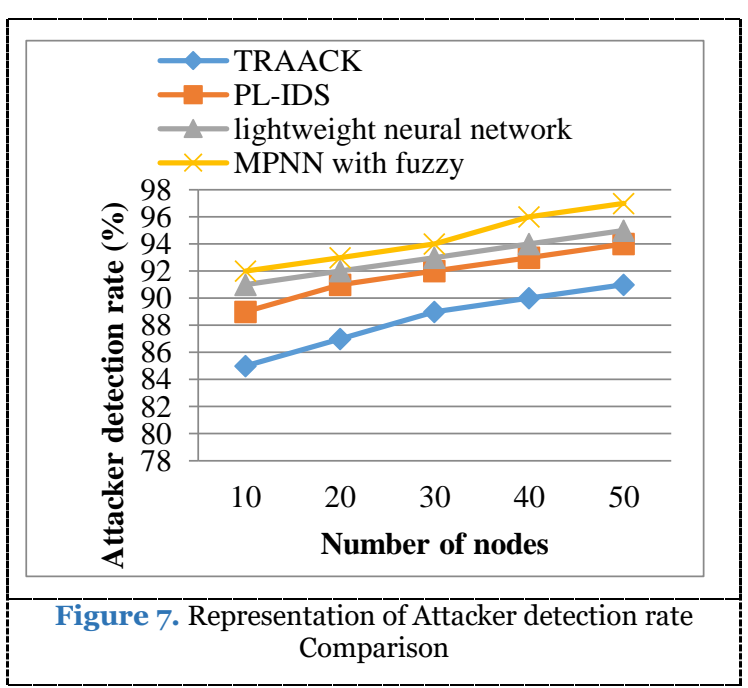

From the above Figure 7, the diagram illustrates the processing time relation for the number of images in the specified datasets. These methods are implemented as TRAACK, PL-IDS, lightweight neural network and MPNN with fuzzy. From this graph, it is known that the proposed MPNN with fuzzy algorithm is higher than the existing algorithms with a high precision rate of $97 \%$ in terms of better attacker classification results. The numerical results of Accuracy Comparison is shown in Table 5.

Table 5. The numerical results of Attacker detection rate Comparison

\begin{tabular}{ccccc}
\hline $\begin{array}{c}\text { Number } \\
\text { of nodes }\end{array}$ & TRAACK & $\begin{array}{c}\text { PL- } \\
\text { IDS }\end{array}$ & $\begin{array}{c}\text { lightweight } \\
\text { neural } \\
\text { network }\end{array}$ & $\begin{array}{c}\text { MPNN } \\
\text { with } \\
\text { fuzzy }\end{array}$ \\
\hline 10 & 85 & 89 & 91 & 92 \\
\hline 20 & 87 & 91 & 92 & 93 \\
\hline 30 & 89 & 92 & 93 & 94 \\
\hline 40 & 90 & 93 & 94 & 96 \\
\hline 50 & 91 & 94 & 95 & 97 \\
\hline V. CONCLUSION AND FUTURE \\
WORK
\end{tabular}

In this work, an IDS against hello flooding, wormholes and Sybil attacks in wireless sensor networks is suggested for wireless sensor networks that are using both anomaly detection and misuse detection to block malicious This system utilizes a Multilayer Perceptron Neural Network based on a fuzzy logic mechanism with a high detection rate and a low false positive rate. The detection mechanism is included in LEACH protocol for cluster-based topology to minimize transmission costs and energy usage, leading to an improvement in network life. The simulation results demonstrate that a proposed MPNN is able to produce high reallife positives and low false positives. This is accomplished with a fuzzy algorithm. In order to evaluate the performance of the proposed research and also encourage biological methods to be integrated into classification algorithms, further analyses are required in the future on this subject.

\section{REFERENCES}

[1]. Sarigiannidis, P., Karapistoli, E., \& Economides, A. A. (2015). Detecting Sybil attacks in wireless sensor networks using UWB ranging-based information. expert systems with applications, 42(21), 7560-7572.

[2]. Patel, R., Pariyani, S., \& Ukani, V. (2011). Energy and throughput analysis of hierarchical routing protocol (LEACH) for wireless sensor network. International Journal of Computer Applications, 20(4), 32-36.

[3]. Sarigiannidis, P., Karapistoli, E., \& Economides, A. A. (2015). Detecting Sybil attacks in wireless sensor networks using UWB ranging-based information. expert systems with applications, 42(21), 7560-7572.

[4]. Kim, G., Lee, S., \& Kim, S. (2014). A novel hybrid intrusion detection method integrating anomaly detection with misuse detection. Expert Systems with Applications, 41(4), 1690-1700.

[5]. Shen, Y., Liu, S., \& Zhang, Z. (2015). Detection of hello flood attack caused by malicious cluster heads on LEACH protocol. International Journal of Advancements in Computing Technology, 7(2), 40.

[6]. Farooqi, A. H., Khan, F. A., Wang, J., \& Lee, S. (2013). A novel intrusion detection framework for wireless sensor networks. Personal and ubiquitous computing, 17(5), 907919.

[7]. Riecker, M., Biedermann, S., El Bansarkhani, R., \& Hollick, M. (2015). Lightweight energy consumption-based intrusion detection system for wireless sensor networks. International Journal of Information Security, 14(2), 155-167.

[8]. Salmon, H. M., De Farias, C. M., Loureiro, P., Pirmez, L., Rossetto, S., Rodrigues, P. H. D. A., ... \& da Costa Carmo, L. F. R. (2013). Intrusion detection system for wireless sensor networks using danger theory immune-inspired techniques. International journal of wireless information networks, 20(1), 39-66.

[9]. Rajeshkumar, G., \& Valluvan, K. R. (2017). An energy aware trust based intrusion detection system with adaptive acknowledgement for wireless sensor network. Wireless Personal Communications, 94(4), 1993-2007.

[10]. Zhang, W., Zhu, S., Tang, J., \& Xiong, N. (2018). A novel trust management scheme based on Dempster-Shafer evidence theory for malicious nodes detection in wireless sensor networks. The Journal of Supercomputing, 74(4), 1779-1801. 
[11]. Ghugar, U., Pradhan, J., Bhoi, S. K., Sahoo, R. R., \& Panda, S. K. (2018). PL-IDS: physical layer trust based intrusion detection system for wireless sensor networks. International Journal of Information Technology, 10(4), 489-494.

[12]. Ravipati, R. D., \& Abualkibash, M. (2019). Intrusion Detection System Classification Using Different Machine Learning Algorithms on KDD-99 and NSL-KDD Datasets-A Review Paper. International Journal of Computer Science \& Information Technology (IJCSIT) Vol, 11.

[13]. Yan, K. Q., Wang, S. C., Wang, S. S., \& Liu, C. W. (2010, July). Hybrid intrusion detection system for enhancing the security of a cluster-based wireless sensor network. In 2010 3 rd international conference on computer science and information technology (Vol. 1, pp. 114-118). IEEE.

Cite this article as: Narmatha C. A New Neural Network-Based Intrusion Detection System for Detecting Malicious Nodes in WSNs. J. Comput. Sci. Intell. Technol. 2020; 1(3): o1-o8. C JCSIT, MNAA PUB WORLD, 2020. 